ARTÍCULO

\title{
El mejoramiento educativo analizado desde los aportes de la sociología de Margaret Archer: un estudio de caso
}

\section{RESUMEN}

Este trabajo aborda el desafío de utilizar un marco analítico sociológico para el estudio del mejoramiento educativo. Se consideró la lógica analítica de la teoría morfogenética de Margaret Archer para estudiar los procesos desarrollados por un conjunto de escuelas chilenas, en el marco de la implementación de una política educativa. Se utilizó la metodología de estudio de casos para la investigación y en este artículo se presenta uno de los casos de estudio. El análisis morfogenético permitió identificar los múltiples constreñimientos a los agentes involucrados con la escuela, de parte de los componentes de la estructura social y cultural. La visibilizadad de estos aspectos, y de la interrelación que tienen entre sí, ayudan a comprender la ausencia de transformaciones positivas en dicho caso.

PALABRAS CLAVE

mejoramiento educativo; teoría sociológica; políticas educativas; teoría morfogenética; Margaret Archer.

Universidade de Chile, Santiago, Chile. 


\title{
THE EDUCATIONAL IMPROVEMENT ANALYZED FROM THE CONTRIBUTIONS OF THE SOCIOLOGY OF MARGARET ARCHER: A CASE STUDY
}

\begin{abstract}
This paper addressed the challenge of using a sociological analytical framework for the study of educational improvement. The analytical logic of Margaret Archer's morphogenetic theory was considered to study the processes developed by a group of Chilean schools, within the context of the implementation of an educational policy. The case study methodology was used for this investigation and this article presents one of the case studies. The morphogenetic analysis allowed to identify the multiple constraints to the agents involved with the school, from the components of the social and cultural structure. The visibility of these aspects, and the interrelation that they have with each other, help to understand the absence of positive transformations in this case.
\end{abstract}

\section{KEYWORDS}

school improvement; sociological theory; educational policy; morphogenetic theory; Margaret Archer.

\section{A MELHORIA EDUCACIONAL ANALISADA A PARTIR DAS CONTRIBUIÇÕES DA SOCIOLOGIA DE MARGARET ARCHER: UM ESTUDO DE CASO}

\section{RESUMO}

Este artigo abordou o desafio de usar uma estrutura analítica sociológica para o estudo da melhoria educacional. A lógica analítica da teoria morfogenética de Margaret Archer foi considerada para estudar os processos desenvolvidos por um grupo de escolas chilenas, no âmbito da implementação de uma política educacional. A metodologia do estudo de caso foi utilizada para essa investigação e este artigo apresenta um dos estudos de caso. A análise morfogenética permitiu identificar as múltiplas restrições aos agentes envolvidos com a escola, por parte dos componentes da estrutura social e cultural. A visibilidade desses aspectos e a inter-relação entre eles ajudam a entender a ausência de transformações positivas nesse caso.

\section{PALAVRAS-CHAVE}

melhoria educacional; teoria sociológica; política educacional; teoria morfogenética; Margaret Archer. 


\section{EL MEJORAMIENTO EDUCATIVO ENTENDIDO COMO CAMBIO SOCIAL}

Los sistemas educativos han ido definiendo como uno de sus propósitos fundamentales de las últimas décadas el mejoramiento educativo (Mourshed, Chijioke y Barber, 2010). Las estrategias orientadas hacia el logro de este objetivo han sido motivo de una profusa discusión y debate. La discusión considera diferentes planos de análisis, incluyendo la pregunta sobre qué se entiende por mejoramiento educativo, cuál es el rol del estado en él, cómo debe organizarse el sistema educativo, de las escuelas y los profesionales de la educación para el logro de ese propósito, cuáles son los tipos de desempeño que dan cuenta del mejoramiento educativo, y cómo se miden, entre otros tópicos que han sido altamente polémicos en la discusión sobre las políticas educativas (Macbeath, 2011).

Pese a la existencia de esta ardua discusión, existe consenso en que los sistemas educacionales deben orientarse hacia el logro de mejoras en su funcionamiento, en el de las escuelas y, sobre todo, hacia la transformación positiva de la formación y aprendizaje de los estudiantes (Bellei et al., 2014; Elmore, 2010). Esto implica que las innovaciones, procesos organizacionales, orientaciones simbólicas y desarrollo de capacidades que estén involucradas en un cambio educativo, solo pueden considerarse eficaces en la medida de que incidan positivamente en el proceso de enseñanza y aprendizaje (Hopkins, 2008; Levin, 2009; Elmore, 2010; Fullan, 2011).

El estudio del mejoramiento educativo se ha enfocado en ganar conocimiento respecto de cómo se produce este proceso de cambio educativo, enfatizando en los procesos, factores y estrategias que pueden incidir en las transformaciones positivas de los sistemas, escuelas y actores educativos (Fullan, 1982, 2002). A este enfoque han contribuido múltiples investigaciones en las últimas décadas, las que han tenido una importante influencia sobre el diseño e implementación de políticas, programas o proyectos educativos (Murillo, 2003; Fullan, 2002; Rincón-Gallardo y Fleisch 2016).

Una de las características más relevantes del enfoque de mejoramiento educativo es su naturaleza procesual, lo que ha propiciado una particular atención a la forma en que se realiza el cambio en educación y no únicamente a sus resultados finales. Anderson et al. (2012) observan que los caminos que puede desarrollar el mejoramiento educativo no siguen necesariamente las mismas vías y que tampoco se dan de una forma necesariamente lineal. Pese a ello, sí se puede constatar que es necesario que exista una ruta o camino hacia el mejoramiento, lo que ha motivado a la generación de múltiples propuestas de etapas o ciclos de mejoramiento (Hallinger y Heck, 2011).

El mejoramiento educativo es un proceso de transformación, y como tal, guarda similitudes con otros procesos de cambio social. La dinámica entre estabilidad y cambio social ha sido objeto de análisis permanente en la historia de la sociología, que, para abordar este fenómeno, ha desarrollado un extenso debate sobre la relación entre estructura y agencia (Aguilar, 2008).

La tensión entre las propuestas analíticas que priorizan al individuo o a la estructura en la explicación de los procesos sociales se ha vuelto crecientemente ineficaz para la comprensión de la complejidad social (Aguilar, 2013). La sociología contemporánea ha intentado avanzar en esta tensión, buscando superar la 
dualidad y reconociendo que los sujetos son influenciados por la estructura, pero que, aun así, que la acción social dispone de ciertos márgenes para los sujetos (Bourdieu, 1991; Giddens, 1995). Archer (2009) avanza en esta proposición de análisis, a través de la noción de "emergencia de lo social", o morfogénesis. Esta considera el vínculo entre estructura y agencia -entendidos como estratos mutuamente irreductibles de la realidad- como una interacción que propicia procesos de estabilidad o cambio en ambas partes. Esta interacción está además fuertemente mediada por el tiempo, además de otros factores, como el contenido y el volumen de la transformación.

La teoría morfogenética propuesta por Archer identifica sus raíces a nivel ontológico en el realismo crítico y la perspectiva analítica relacional, propuesta por Roy Bhaskar (2003), que destaca que las personas son influidas por las relaciones sociales, pero que éstas, a su vez, son el resultado de la actividad humana. Es decir, la naturaleza de los agentes es social, pero estos a su vez reflexionan, razona y eligen en el contexto social concreto en el que se desarrolla su vida (Sayer, 1992).

La teoría morfogenética de Archer (2009) comprende que los agentes están social y culturalmente condicionados, es decir, su acción se encuentra constreñida por su rol, posición, características sociales y normas. Sin embargo, la noción de emergencia asume también que los agentes -enmarcados además en los procesos culturales- disponen de espacios que les permiten orientar su vida según sus objetivos, lo que favorece la explicación de la transformación social en el tiempo.

Archer $(2007 \mathrm{a}, 2009)$ utiliza el concepto de emergencia para analizar cómo se produce el cambio social, así como también las condiciones que se requieren para su transformación o mantención. Según indica esta autora, en la sociedad, la estructura y la agencia poseen propiedades que les son propias y autónomas. Ambos estratos se relacionan entre sí, pero a través de una secuencia temporal, donde la primera precede a la segunda. La interrelación de ambos componentes permite que los atributos de ambas generen un nivel emergente, es decir, atributos o características nuevas o modificadas en cada estrato. La dinámica de interacción es definida como ciclo morfogenético.

Es posible identificar elementos comunes entre la lógica analítica del estudio del mejoramiento educativo y la propuesta morfogenética de Archer. Ambas coinciden en la revisión de un proceso de estabilidad o cambio, en el que participan agentes, instituciones y un contexto. Esta reflexión inicial motiva a hipotetizar que el reconocimiento de las características y dinámicas de estructura y agencia, puede ayudar a identificar o profundizar en el conocimiento de factores, condiciones y contextos que facilitan o que dificulten el mejoramiento educativo.

Considerando estos desafíos analíticos del estudio del mejoramiento educativo y la forma en que se asemeja a los planteamientos sociológicos respecto del cambio social, en este trabajo se planteó la posibilidad de recurrir al aporte del análisis morfogenético, como una forma de profundizar en el estudio del mejoramiento educativo. La expectativa de este ejercicio es identificar claves o lógicas analíticas del estudio del cambio social, que puedan ayudar a comprender desde otra perspectiva el mejoramiento educativo. Los aprendizajes de este ejercicio pueden ser de utilidad para profundizar en formas de analizar los procesos que 
caracterizan la mejora, pero también a reconocer elementos que pueden ser considerados desde las políticas educativas para generar impulsos efectivos orientados al objetivo del mejoramiento.

\section{EL ENFOQUE MORFOGENÉTICO Y SU APORTE A LA COMPRENSIÓN DEL CAMBIO SOCIAL}

Como se anticipó, ciclo morfogenético es el nombre del modelo analítico de la interacción entre agencia y estructura que puede generar una transformación social, propuesto por Archer (2007b, 2009). El ciclo morfogenético da cuenta del proceso de cambio social (o estabilidad), producto de la dinámica de interacción entre agencia y estructura, o entre diferentes grupos sociales, y consta de tres partes: condicionamiento estructural y cultural, interacción social y elaboración estructural. El condicionamiento estructural y cultural, es el primer momento en el ciclo. Se entiende que las propiedades sistémicas de una sociedad son consecuencias de acciones pasadas y, a la vez, se convierten en el marco que limita y permite la acción de los agentes. La estructura cuenta con propiedades condicionantes de carácter social y cultural. Las primeras tienen una dependencia directa de recursos materiales, tanto físicos como humanos. Las segundas, están relacionadas con el mundo de las ideas y de las normas sociales.

La interacción social corresponde a la relación que los agentes establecen con la estructura y con otros agentes por medio de la acción. La interacción es estructuralmente condicionada, pero no estructuralmente determinada. El condicionamiento incorpora el reconocimiento de la creatividad de intereses y la capacidad de dar respuestas innovadoras frente a los constreñimientos, así como al autosacrificio de los intereses heredados.

La elaboración estructural es una consecuencia fundamentalmente no deseada (Archer, 2009, p. 138). La modificación de las estructuras previas y la introducción de otras nuevas es el producto combinado de resultados perseguidos por grupos diferentes en forma simultánea en el segundo momento del ciclo. Este punto también es el inicio de un nuevo ciclo morfogenético, es decir, de un nuevo proceso de interacción entre la estructura y la agencia.

\section{LA DINÁMICA DEL CICLO MORFOGENÉTICO}

De acuerdo con Archer (2009), el ciclo morfogenético es un proceso en el que puede darse o no el cambio social, vale decir, puede haber morfogénesis o morfoestásis, es decir, potencialmente pueden ocurrir o no suceder los cambios esperados por un grupo de actores sociales.

Las etapas de ciclo morfogenético se desarrollan dentro de cada estrato (estructura, cultura y agencia) y la interrelación entre estos en su conjunto permite ver el resultado del proceso morfogenético.

Respecto de las condiciones de la estructura y la cultura, los poderes causales de la mantención o el cambio operan bajo condiciones específicas. La estructura 
y la cultura ejercen influencias condicionales sobre la agencia, sin determinarla, puesto que ésta es portadora de poderes emergentes. Esto implica que el condicionamiento sociocultural solo ejerce sus efectos en las personas y es eficaz a través de las personas.

En el contexto descrito, las transformaciones se dan producto de la confluencia de dos o más conjuntos de poderes, cuya combinación genera una modificación del statu quo, mientras que la reproducción (morfoestásis) ocurre cuando los poderes agenciales se han tornado poco efectivos para enfrentar las influencias estructurales. La combinación de estos poderes puede darse en una lógica de integración o complementariedad o alta integración, o en una de baja integración o incompatibilidad. Asimismo, también será relevante comprender si la interacción de los factores es carácter de necesario (relaciones estables y compatibles) o de contingente (producidas por influencias externas), para profundizar en la identificación de transformaciones o estabilidad en esos casos. El resultado de esa interacción, entonces, tiene efecto en el resultado del proceso morfogenético.

En la teoría de Archer $(2000,2007 b)$ la agencia tiene un papel fundamental en el proceso morfogenético, cuya acción posee un margen de maniobra con relación a los determinantes de la estructura. El nivel posicional de los agentes está relacionado con la existencia de intereses creados, es decir, características objetivas de las situaciones que viven los agentes y que los predisponen hacia cursos de acción o estilos de vida. Los agentes pueden actuar en contra de esos intereses, pero deben asumir los costos que ello implicará, puesto que los intereses creados tienen beneficios para la satisfacción de necesidades que no están igualmente distribuidas en la sociedad. Archer denomina a estas consecuencias como costos de oportunidad.

Del juego mutuo entre estructura y agencia, se avanza por el ciclo morfogenético, desde la etapa de condicionamiento estructural, pasando por la de interacción social y luego a la de elaboración social. Cuando la integración social y sistémica están alineadas, hay tendencia a la morfoestásis. Mientras, una débil integración de ambas en un momento da origen a la morfogénesis.

\section{¿PUEDE LA TEORÍA MORFOGENÉTICA AYUDAR A COMPRENDER EL MEJORAMIENTO EDUCATIVO?}

Ciertamente, la teoría morfogenética ha sido creada para analizar grandes procesos sociales, los que se extienden por extensos períodos de tiempo, lo que implica que es una perspectiva que no se diseñó originalmente para analizar fenómenos específicos, aspecto que le quita cierta capacidad de comprensión más pragmática. Por su parte, los procesos analizados por la perspectiva de mejoramiento educativo a la que se ha hecho alusión -más acotados y pragmáticos-, sobre todo en contextos de alta necesidad de cambio, requieren de transformaciones que son mucho más cortas en el tiempo.

Sin embargo, y sin interés de homologar ambos procesos, se considera que el estudio de los componentes y la forma de interacción entre condicionantes estructurales y culturales, puede proporcionar antecedentes que ayuden a comprender los procesos de mejoramiento educativo. 
La lógica del ciclo morfogenético da cuenta de un análisis histórico de un proceso de transformación, es decir, se concentra en su implementación. Desde en campo educativo, ello puede interpretarse desde la investigación, como el estudio de la interacción entre las propiedades de la estructura social y cultural (representadas en este caso por el sistema educativo, el contexto social de la escuela y sus normas fundamentales) con los agentes (en este caso los actores individuales y colectivos en las comunidades educativas).

Así, dando cuenta del juego mutuo entre la estructura y agencia podría avanzarse en la identificación más particularizada de la dinámica del mejoramiento educativo, de su estabilidad, o incluso, de su desmejoramiento. Lo anterior, además puede ser de utilidad como insumo para las estrategias que se orientan a generar impulsos para el mejoramiento educativo, dado el conocimiento desde las que éstas pueden fundarse.

\section{METODOLOGÍA}

Se diseñó una investigación que, utilizando el marco referencial ya mencionado, buscó analizar las características que tenía el proceso mejoramiento educativo en diferentes establecimientos educacionales, en el marco del ciclo de implementación de una importante política educativa chilena. En particular, se consideró la política llamada Subvención Escolar Preferencial (SEP), que implica una importante inyección de recursos para las escuelas, que debe ser utilizada en la ejecución de un Plan de Mejoramiento Educativo (PME), donde debiera expresarse la estrategia de mejoramiento de la institución. El primer ciclo de ejecución del PME se extendió por cinco años y fue este período en el que se enmarcó el estudio del mejoramiento educativo en las escuelas que fueron objeto de la investigación.

Manteniendo el foco en la utilización de la lógica analítica del análisis morfogenético de Archer, se diseñó un estudio de carácter retrospectivo que se orientó a analizar los procesos de estabilidad y cambio en los procesos y resultados educativos de las escuelas seleccionadas, así como los factores que los explican.

Utilizando las etapas del ciclo morfogenético, y aplicándolas al ciclo de la ley SEP, se definieron tres etapas teóricas, las que se trabajaron en un orden diferente al referido por Archer, debido al carácter retrospectivo de la investigación:

- Situación actual: elaboración estructural. Corresponde al estado final del proceso de implementación del PME SEP, luego de los cinco años de implementación de esta política. La hipótesis de la SEP era que, durante este período habrían ocurrido cambios positivos en aspectos organizacionales, de prácticas y logros académicos en el establecimiento;

- Condicionamiento estructural. El estado actual de implementación se contrastó con su estado inicial, ocurrido al inicio del ciclo de mejoramiento SEP (cinco años antes). Se puso énfasis en identificar la medida en que los involucrados vivieron u observaron el condicionamiento de los componentes estructurales sociales y culturales de los procesos desarrollados por el establecimiento en los primeros momentos de implementación de la SEP; 
- Interacción social. El tercer elemento de interés para el análisis de la implementación es el que permite describir los procesos que ocurren durante el desarrollo del ciclo SEP. En esta línea, se esperó identificar y explicar cómo ha operado la interrelación entre los agentes con los condicionantes estructurales, para identificar qué aspectos favorecen la reproducción del diseño original y qué otros elementos se han ido transformando en el tiempo.

La investigación adquirió la estructura metodológica de un estudio de casos. Fueron seleccionadas 8 escuelas públicas chilenas, que representan el cruce entre dos variables fundamentales para ese sistema educativo: el nivel de desempeño de la escuela y el nivel de vulnerabilidad socioeconómica de sus estudiantes. En el caso del desempeño se consideraron escuelas clasificadas en las tres categorías definidas por la institucionalidad educativa: autónomas (presentan estudiantes con altos resultados educativos), emergentes (no alcanzan consistentemente esos resultados) y en recuperación (con resultados reiteradamente insuficientes) (Agencia de Calidad de la Educación, 2014). Respecto de la vulnerabilidad, se consideró el porcentaje de estudiantes identificados por el estado como "prioritarios”, debido a sus características socioeconómicas. Se identificaron escuelas que estuvieran por sobre y bajo el porcentaje promedio de estudiantes prioritarios en las tres categorías de escuelas ya mencionadas. Finalmente, se agregaron dos escuelas rurales, que por su número de estudiantes participan de forma más tangencial en la política educativa.

Para este artículo, se presentará uno de estos casos, para poder describir en profundidad la manera en que puede analizarse sus transformaciones a partir de la teoría morfogenética.

El trabajo de producción de información consistió en la realización de seis entrevistas en profundidad o grupos focales a representantes de los organismos involucrados en la implementación del PME SEP, vinculados a cada escuela analizada: directivos de la escuela (director y jefe técnico), representantes del "sostenedor" (se trata de la institución que administra y representa legalmente a la escuela a nivel local), Supervisor del Ministerio de Educación que orienta y trabaja con la escuela, apoderados (padres, madres o cuidadores) y asistencia técnica privada (instituciones que son contratadas para asesoras a las escuelas). Lo que se buscó en las entrevistas fue identificar qué características tenía la institución educativa en cada una de las tres etapas teóricas descritas, cuál era el rol particular, facilidades y dificultades que tenía el actor entrevistado para el desempeño de su rol y qué vínculos y aportes tenía la SEP y el PME en la escuela en cada período analizado.

Las entrevistas se realizaron siguiendo la propuesta metodológica de la misma Archer (2007a) quien indica que son los agentes quienes mejor pueden dar cuenta de la interacción entre estructura y agencia y de los elementos que constriñen y favorecen su acción, en este caso, de la acción de la escuela.

Las entrevistas fueron organizadas, de forma que, para cada etapa teórica del ciclo del PME, los sujetos describieran las características de la escuela y de su propio rol, considerando un conjunto de factores clave asociados al mejoramien- 
to educativo, que pudieran relacionarse con los conceptos centrales de la teoría morfogenética. Las entrevistas terminaron de aplicarse en el año 2014 y fueron transcritas y codificadas, considerando las tres etapas del ciclo morfogenético ya descritas. Se consideraron en el análisis aspectos relacionados a la ley SEP, a los factores condicionantes sociales y culturales de la acción de la escuela y de los involucrados, las características de la agencia y el tipo de relación entre los involucrados en cada una de las etapas mencionadas. El análisis de dicha información se realizó por medio de la metodología de análisis de la Grounded Theory (Strauss y Corbin, 1990).

Adicionalmente, se consideró el análisis de información secundaria de la escuela, particularmente con relación a sus resultados en la medición de Simce, así como en su matrícula. Estos dos indicadores -fundamentales para dar cuenta de resultados escolares en Chile- se utilizaron para contrastar la información provista por los entrevistados, de manera de brindar mayor estabilidad a la evaluación del proceso de mejoramiento de las escuelas, en cada etapa analizada.

Lo que se buscó fue conformar un esquema explicativo que diera cuenta de la implementación del PME SEP durante el ciclo de 5 años, y que identificara su aporte al proceso de mejoramiento de la escuela. Este esquema está conformado por los factores que, incidieron desde la estructura o la agencia en el proceso de mejoramiento de la escuela, en el marco de la implementación del PME SEP.

\section{RESULTADOS}

\section{DESCRIPCIÓN DEL CASO DE ESTUDIO}

Antes de exponer el caso del proceso de mejoramiento de una escuela en particular, es relevante ofrecer una contextualización sobre las características del sistema educativo chileno, debido a que reporta elementos estructurales relevantes para el análisis morfogenético.

El sistema escolar chileno es de carácter mixto, es decir, existen proveedores (escuelas) públicos, privados y "particulares subvencionados", estos últimos es de propiedad privada, pero financiados con recursos públicos. El financiamiento de las instituciones públicas y particulares subvencionadas opera principalmente a través de la subvención escolar, es decir, un monto de recursos que los establecimientos reciben por cada alumno que educan y que asiste a la escuela. Hasta antes de la ley de Subvención Escolar Preferencial (SEP), la principal fuente de financiamiento era la subvención por asistencia escolar.

Luego, se incluyó la SEP como un mecanismo que busca compensar ingresos de las instituciones, sobre la base de los alumnos vulnerables que recibe. Es decir, destina un conjunto de recursos por cada estudiante vulnerable que se educa en una escuela.

Pese a la relevancia de la SEP, este mecanismo no modificó la base del funcionamiento del sistema de financiamiento, en base a la lógica de vouchers. Este componente, así como otros, dan cuenta de un sistema que considera y utiliza una lógica de mercado, en que las instituciones debieran competir por la matrícula 
y los apoderados, escoger sobre la base de métodos racionales la mejor institución para que se eduquen sus pupilos, sobre la base de información provista por el mismo sistema (Bellei, Valenzuela y de los Ríos, 2010).

La incorporación de los mecanismos de mercado y el proceso de municipalización de la educación, ambos implementados durante el período de dictadura cívico-militar en Chile, tuvo profundos efectos negativos en las condiciones para el desarrollo de la educación pública en Chile, la que pasó de ser mayoritaria en cuanto a su matrícula, a ser superada en ese aspecto por las instituciones particulares subvencionadas y a profundizar la estratificación socioeconómica de la educación chilena (Bellei, 2013).

A continuación, se presentará la descripción de uno de los casos de estudio en que se aplicaron los procesos de codificación mencionados anteriormente Este relato da cuenta de las características de una escuela en particular y de su proceso de mejoramiento desarrollado en el ciclo de cinco años, en el marco de la implementación del ciclo de mejoramiento del PME SEP.

\section{CASO: "ESCUELA EN RECUPERACIÓN CON ALTO PORCENTAJE DE VULNERABILIDAD". DESCRIPCIÓN GENERAL DEL CONTEXTO SOCIOEDUCATIVO DE LA ESCUELA}

El caso presentado refiere a una escuela queda ubicada en una de las comunas más pobladas de la capital del país y se encuentra en medio de una villa de viviendas sociales, donde viven personas en condiciones de alta vulnerabilidad, ya sea por pobreza u otros factores de riesgo social. Asisten estudiantes provenientes de esa villa. Al momento de finalizar el proceso de levantamiento de información, el Índice de Vulnerabilidad Escolar de este liceo -indicador elaborado por el Estado- era del $92,55 \%$, es decir, prácticamente la totalidad de los alumnos son considerados como vulnerables, en términos socioeconómicos. Este porcentaje se mantuvo estable en el período analizado y con posterioridad a él. En su entorno se reconocen acciones de conflicto y delincuencia y la escuela ha sido víctima de robos de parte de sus propios vecinos. El año de finalización de levantamiento de información de esta investigación, la comuna en que se encuentra la escuela estaba en el tercer lugar en un índice "socio delictual" en su región, de acuerdo con una asociación de municipios del país (AMUCH, 2014).

El establecimiento tiene alrededor de 17 años de historia y se conformó inicialmente con personas que provenían de territorios rurales y que quedaron desempleados con el cambio de residencia, pero luego el perfil fue eminentemente urbano.

A continuación, se relatará el proceso de mejoramiento de la escuela desde la perspectiva morfogenética, siguiendo los tres momentos que se consideraron en el estudio retrospectivo.

\section{MOMENTO 1: INICIO DEL PERÍODO}

Desde su origen, este establecimiento operó considerando débiles expectativas sobre los aprendizajes de sus estudiantes, de parte de los involucrados, lo que a juicio de los entrevistados, estaba influido por el entorno en que este se encuentra 
ubicado. Así, la institución adquirió un perfil más social que educativo, es decir, más como un espacio de contención y protección frente a la vulnerabilidad, que como un lugar de desarrollo de los aprendizajes.

Nosotros nos relacionamos con el Gobierno en terreno, por parte de la Gobernación, y como existe de que muchas familias no tienen un sueldo como corresponde, se ofreció corte de pelo, manicure [...] es lo que se pretendió en ese enganche que se sintiera la matrícula de este colegio. (Apoderada escuela)

En este período, el sostenedor, la escuela y los apoderados buscaron alternativas para educarse en un contexto de pobreza y deprivación, pero no profundizaron en el diseño de un proyecto educativo particular. Se reconoce que no se desarrollaron altos niveles de organización interna, ni de delimitación de roles y funciones. El sostenedor, por su parte, tenía una alta injerencia en la toma de decisiones y en las definiciones relacionadas con el personal.

Además, se advierten grandes dificultades en el clima interno de la escuela, lo que involucra a los profesores y los alumnos, y se describe que la institución no ha logrado funcionar de manera normalizada, es decir, que no puede desarrollar de manera regular procesos básicos de una escuela. Junto con ello, se asume debilidad en el trabajo en equipo y la colaboración entre los funcionarios de la escuela.

Nosotros, con mi compañera y solicitamos ayuda al equipo de convivencia escolar de la Secretaría Ministerial de Educación. Ellos vinieron a la escuela [...] $\mathrm{Su}$ diagnóstico fue que es una escuela que tiene un ambiente bastante enfermo, y para eso necesitaban trabajar muchísimo, hay que hacer un trabajo de reparación, es un trabajo que no es tan fácil ni tan corto. (Representante Ministerio de Educación)

E1 Ministerio de Educación ha estado presente en la escuela desde su fundación, a través de labores de supervisión y apoyo técnico. En ese contexto, el sostenedor suscribió a la SEP, cuyos ingresos fueron administrados con desorden por la escuela, identificándose -por parte de los entrevistados-altas dosis de despilfarro. Pese a ello, parte de los actores declaran la importancia que pudo tener para la escuela la llegada de los recursos de esta subvención y que deba implementarse un plan de mejoramiento, pese a que su implementación haya sido defectuosa.

Y con un montón de recursos, o sea con plata, con todo este montón de recursos no han hecho nada. Se llenaron de personal, psicólogos, fonoaudiólogos, profesores y no tienen capacidad para reclutar personas. [...] El profesor titular, contando con un profesor asistente, habla por teléfono, llegaba atrasado y el otro le hacía en trabajo. (Sostenedor)

Se evalúa que, en dicho período, el sostenedor no brindó suficiente soporte y orientaciones que apoyen la toma de decisiones internas de la institución. Al contrario, se cuestiona la existencia de malas decisiones, asociadas a acciones para el 
mejoramiento de la escuela, elección de equipos directivos de la institución, o sobre el uso de los recursos que disponía la escuela.

Con relación a las competencias de los actores, se identifica un conjunto de factores que constriñeron la acción y la mejora de la institución. Esos elementos estaban dentro de la escuela, considerando la de visión estratégica de parte de la directora, debilidades técnicas y de enseñanza de parte de los profesores, y en general de la labor técnica de las personas que se desempeñan en el liceo.

Ahí ocurrió un problema grande, que la población, o sea, que la dotación era, no quiero juzgar, pero en realidad no tenía, primer lugar, no tenían currículum para atender a esa vulnerabilidad a ese tipo de perfil, o sea, la escuela en si era vulnerable, porque la escuela no tenía herramientas técnicas, salvo excepciones, de atender ese perfil. (Sostenedor)

Por su parte, los actores involucrados destacan que existiría una resistencia o falta de interés en cumplir con el rol que a cada uno lo compete y falta de expectativas en la escuela, lo que dificultaría la acción de los demás y de la propia escuela. A ello, se suma una baja efectividad de las iniciativas de estos agentes, en la puesta en marcha de acciones para la mejora.

Este período está caracterizado por bajos resultados educativos del establecimiento. En la medición nacional Simce, obtuvo un promedio de 215 puntos en cuarto básico, es decir en el cuartil inferior de la distribución de resultados. Mientras, su matrícula era de 750 estudiantes. Esta cifra será relevante cuando se establezca una comparación con los siguientes momentos de análisis.

\section{MOMENTO 2}

El segundo período de análisis, se caracterizó por una profundización de las dificultades evidenciadas en el momento anterior, en un contexto socioeducativo que mantuvo sus complejos atributos. Por lo general, cada uno de los aspectos señalados anteriormente se mantuvieron o agravaron en este período, a juicio de los entrevistados.

Un equipo directivo poco sólido, donde el inspector general tenía que hacer muchas cosas que no correspondían al inspector general, donde la jefa técnica tenía que asumir muchas responsabilidades de inspectora general. Entonces, qué es lo que pasa: el trabajo técnico era el último eslabón, no porque sea malo, sino porque el trabajo de contención del colegio a diario es muy flerte. (Directora)

Al complejo clima educativo, en este período se sumó el escalamiento de problemas de convivencia interna, en una institución que seguía sin lograr la normalización. Los entrevistados que no trabajan directamente en la escuela, hablan de que, en este período, los docentes terminaron siendo dominados por un sentimiento de bajas expectativas y de una actitud reticente y poco favorable a su propia mejora. A este escenario, se suma la continua rotación de personas en cargos directivos. 
Entonces nosotros llegábamos a la escuela, quedamos en un compromiso para la siguiente visita y entonces... "Oye ustedes quedaron de hacer la reunión de análisis de resultados"; "Ah no si no tuvimos tiempo, que problemas porque llovió porque no sé, porque nos faltó el profesor porque tuvimos que estar remplazándolo...” Múltiples razones que tuvieron en este tiempo... (Representante Ministerio)

En esta situación, junto a la dinámica interna, influyó la existencia de serios problemas entre la escuela y el sostenedor por motivos laborales, específicamente por cesación de remuneraciones y de pago para la seguridad laboral, asociados a una crisis financiera de este organismo.

En paralelo, el establecimiento empezó a notar que, luego de los problemas de con el uso de recursos SEP descritos en la etapa anterior, el sostenedor quitó autonomía a la escuela para el uso de esos recursos. De esta forma, el sostenedor concentró el control sobre el gasto de recursos y solo una parte minoritaria de esos ingresos fue efectivamente dirigida por la escuela.

Las corporaciones [sostenedores] como están tan quebradas [...] Entonces, las platas no van al proyecto. Si el sostenedor tiene con dos horas semanales, no hay nada que hacer y si el trabajo son cuatro horas y no han llegado recursos, no se pueden desarrollar en un cien por ciento como se esperaba. (Directora)

En este escenario, la escuela tampoco encontró el rumbo en materia pedagógica, lo que tuvo consecuencia que fuera clasificado en la categoría "en recuperación" (el peor nivel en la clasificación de escuelas), a lo que se sumó la disminución progresiva de su matrícula. Esto estuvo asociado a una disminución del puntaje Simce, que descendió a 211 puntos en promedio. La matrícula también descendió en alrededor de 140 alumnos en este período, respecto del período anterior.

La presencia de la asesoría ministerial se intensificó en este período. Sin embargo, desde el punto de vista de los representantes del Ministerio, no se logró involucrar a los docentes ni a sus directivos en un proceso de mejoramiento.

En ese contexto, los apoderados mantuvieron un foco sobre la escuela más orientado hacia el desarrollo social que educativo, sin manifestar diferencias en las expectativas de aprendizaje de los estudiantes, en relación con el período anterior.

De esta forma, persistió la percepción de bajo alcance de la agencia en la modificación del escenario descrito.

\section{MOMENTO 3}

El período final de análisis en la historia de esta institución, se caracterizó por la mantención de la realidad descrita en los períodos anteriores, tanto en términos de las características del contexto de las escuelas, como en sus procesos internos y logros educativos.

En esta etapa se produjo una huelga de profesores -que se prolongó por un mes-, debido a sus problemas laborales con el sostenedor. A ello, se agregó el 
término de contrato de otro director de la escuela, quien no finalizó nuevamente su período, asumiendo una directora interina, que solo pudo mantenerse un año en la gestión. La dinámica organizacional de la escuela no varió durante esta última gestión, sino que más bien se complicó, debido a que la directora fue víctima de agresiones de parte de algunos profesores.

Nunca me imaginé que todo sería tan difícil, todo lo que me imaginé quedó corto $[\ldots]$ me frustra el no poder haber desarrollado un clima laboral que permita un buen clima escolar y, por lo tanto, desarrollar un proceso de aprendizaje de mejor forma. Hay profesores que no quieren entender, se mimetizaron con el medio, son groseros. (Directora)

El sostenedor mantuvo en este período el estilo de gestión de los recursos de la institución. Por ello, la escuela definió una planificación estratégica que no pudo implementar por falta de recursos y de autonomía. A ello, se fueron agregando dudas, cuestionamientos y suspicacias sobre los usos que hacía el sostenedor de los recursos pertenecientes al establecimiento.

El Ministerio de Educación siguió focalizando su trabajo en esta institución, implementándose una visita de asesoría que opera de manera regular. Pese a ello, los participantes estiman que ésta siguió siendo poco efectiva, en cuanto a sus logros en las dinámicas internas, la gestión de la escuela y sus resultados:

El año anterior yo diría que fue un año bastante mejor [que el actual] en términos de que ya el programa había entrado, había entrado y estaba diríamos que en fase de instalación. Yo diría que este año volvíamos a estar en la misma etapa de instalación. (Representante Ministerio de Educación)

Como era de esperar, en base a este relato, los actores perciben que no ocurrieron grandes cambios en este período, salvo los intentos fallidos, provocados por motivación o interés de algunos de los participantes de la escuela para generar mejoras. Es el caso de la directora y la jefa de UTP del colegio, que son destacados por la propia directora y Ministerio, como personas que buscaron, sin éxito, usar sus capacidades personales para incidir en la dinámica de la escuela y el compromiso de los involucrados.

Este colegio es un colegio con muchas problemáticas. La última directora, a lo menos, hizo mucho empeño en hacer bien las cosas, pero tuvo muchos problemas porque no logró validarse con los equipos. Además, sabes que la ley viene asociada a dineros, y esos dineros ellos no los recibieron, porque la corporación está muy complicada con los temas económicos [...] Yo diría que esta es una escuela muy desencantada. (Representante Ministerio de Educación)

Este última etapa estuvo marcada por una nueva disminución en el puntaje Simce, que llegó a un promedio de 200 puntos en Cuarto Básico. La matrícula descendió también, en alrededor de 100 estudiantes, respecto del momento anterior. 


\section{ANÁLISIS DE LA INFORMACIÓN: HACIA UN ANÁLISIS MORFOGENÉTICO DEL PROCESO DE MEJORAMIENTO DEL CASO ESTUDIADO}

\section{ROL DE LOS FACTORES DE LA ESTRUCTURA Y LA AGENCIA EN EL PROCESO}

A continuación, se analizará la vinculación entre los factores relacionados con la estructura y agencia y la interacción entre estos estratos, evaluando si, desde la perspectiva de los involucrados, esta relación favoreció o constriñó la acción de los mismos actores y de la escuela y con ello de qué forma afectó su proceso de mejoramiento.

Con relación a los factores condicionantes de tipo estructural, los resultados académicos de la escuela no se convirtieron en un factor que favoreciera el mejoramiento de la escuela. Al contrario, la existencia de negativos resultados fue considerado como un factor que constriñó el proceso de mejora, considerando un impacto negativo en las expectativas del entorno respecto de la escuela.

Respecto de las condiciones técnicas para el funcionamiento de la escuela, se identificó que las grandes debilidades y carencias descritas, a nivel de funcionamiento del sostenedor y de la escuela, afectaron en un constreñimiento de las oportunidades para la mejora institucional. Lo propio se observó en relación con la presencia de recursos humanos, financieros y contratación de servicios, cuya disponibilidad fue débil durante todo el período.

Respecto de las políticas educativas (especialmente la SEP), su implementación implicó la generación de un conjunto de expectativas de mejoramiento, que finalmente se fueron deshaciendo con el tiempo. Esto fue especialmente claro respecto del financiamiento que entrega esta política. Sin embargo, algunos de los esfuerzos más relevantes para la mejora escolar se hicieron en el marco del trabajo que realizó el Ministerio de Educación en esa escuela. Como pudo observarse, la evaluación final de este apoyo tampoco fue positiva.

La gestión de la institución sostenedora y la relación entre ésta y la institución, fueron otro elemento que constriñó las posibilidades de mejora, debido a los serios problemas de entrega y uso de los recursos, a la ausencia de procesos relevantes de apoyo técnico y la poca autonomía que se entregó a la escuela.

Todos los elementos mencionados se enmarcan en un conjunto de características históricas (heredadas) de tipo geográfico, social y económico del entorno y las características de los estudiantes y sus familias, los que desafiaban aún más los procesos educativos de la institución.

Con relación a los factores estructurales de tipo cultural, en este caso, propios de la gestión de atributos culturales de la esta escuela, específicamente respecto del liderazgo pedagógico del sostenedor, pudo identificarse la ausencia de un liderazgo pedagógico y el predominio de una relación de control administrativo. Nuevamente, este factor incidió de manera negativa en el proceso de mejora.

Con relación a la imagen y expectativas existentes respecto de la escuela, se identificó la presencia y mantención de un fuerte estigma, lo que se profundizó al ser catalogada en la categoría "en recuperación”. Este aspecto tuvo efectos concretos en la disminución de la autonomía de la institución. 
Respecto del factor liderazgo pedagógico del director, en el período analizado este factor se observó en mayor medida por su ausencia, incluso cuando la última directora intentó establecer ajustes en la gestión de la escuela. En este caso, los esfuerzos no fueron fructíferos.

Con relación a los factores que se vinculan con la agencia, específicamente sobe las características de los apoderados y su relación con la escuela, se observó un foco no académico de los apoderados respecto de la institución, lo que, a juicio de los entrevistados, potenció la ausencia de un proyecto educativo.

Respecto de las condiciones personales del director y su equipo, el relato analizado dio cuenta de que, en algunos momentos, se contó con personas que podrían tener condiciones positivas para afrontar los desafíos de la mejora. Sin embargo, fracasaron en sus intentos, dada la complejidad del escenario descrito. Lo propio ocurre si se analiza la gestión del equipo directivo, que no logró introducir cambios concretos en los procesos institucionales y por ello, no incidió mayormente en el proceso de mejora.

Con relación a las habilidades y disposición de los docentes y funcionarios de la escuela, se identificó un importante grado de resistencia a la modificación del statu quo, afectando negativamente la implementación de procesos de transformación.

\section{ANÁLISIS MORFOGENÉTICO DEL PROCESO DE MEJORAMIENTO EDUCATIVO}

Luego del análisis individual de los factores asociados al proceso de la escuela, y siguiendo la teoría de Archer, se describirán las características del proceso morfogenético de cada estrato, por separado (agencia, estructura social y estructura cultual). Luego, se avanzará a integrar estos aspectos en el análisis, a partir del uso de los conceptos de intereses creados y nivel posicional descritos por Archer (2007a, 2009), que justamente dan cuenta de la acción de los agentes en un contexto de condicionamiento estructural. Finalmente, con los antecedentes descritos, se evaluarán de manera general los procesos morfogenéticos o morfoestáticos en el conjunto de los casos de estudio.

\section{MORFOGÉNESIS DE LA AGENCIA}

En términos teóricos, cumplen con la condición de agente en el proceso descrito el equipo directivo, los grupos de profesores, el sostenedor, en cuanto asociación de personas que gestionan la educación municipal, el centro de padre y los supervisores en terreno del Ministerio de Educación.

En este caso particular, el equipo directivo pudo abocarse dificultosamente a un rol particular, como es la organización administrativa de la escuela. Mientras, el sostenedor, se orientó preferentemente al control financiero y administrativo. Es decir, ambos agentes no abordaron de manera relevante procesos de liderazgo o de gestión orientadas por un proyecto educativo. Por su parte, los apoderados ejecutaron permanentemente un rol de "beneficiarios" de las políticas sociales que se implementaban a través de la escuela. A su vez, los 
docentes mantuvieron un rol de enseñanza, considerando la estructura inicial del establecimiento y de manera independiente o separada de los procesos definidos por el equipo directivo. Mientras, el equipo territorial del Ministerio de Educación asumió una labor orientada a buscar dotar de sentido pedagógico y organización educativa a la institución.

Archer (2009) menciona que la agencia vive su propio proceso morfogenético. Luego de la etapa de condicionamiento estructural, se transita por medio de los intereses creados a la de interacción social, y el paso de esta etapa a la de elaboración estructural (cambio social) requiere del poder y del intercambio. En este caso en particular, ninguno de los agentes modificó de manera relevante sus características y roles principales, ni tampoco logró vincularse o afectar los de los otros agentes involucrados lo que puede observarse con la presencia variada e incompatible de los roles que ejecutaron. Es decir, los agentes se mantendrían en una situación de condicionamiento estructural, usando los términos de Archer.

\section{MORFOGÉNESIS DE LA ESTRUCTURA}

Respecto del análisis de las estructuras y de su disposición e integración previa al período analizado, en el caso presentado se identificó la presencia de Ministerio de Educación, sostenedor y escuela como las instituciones que ya formaban parte de la estructura que condicionaba la escuela, las que operaban en base a recursos financieros, educativos, de normas y procedimientos de monitoreo y control.

De los discursos analizados se distinguió una lógica más bien vertical de integración entre estos elementos, es decir, las definiciones del sistema educativo llegaban a las escuelas a través del sostenedor, o las políticas del sostenedor "bajaban" hacia la escuela.

Respecto de las propiedades estructurales de la cultura, las escuelas ya participaban previamente del Sistema nacional de medición de aprendizajes (Simce) y de la lógica y sentido de política educativa a la que ésta aporta, como indicador relevante de calidad educativa. Mientras, las directrices educativas de Mineduc -en cuanto a lógicas de funcionamiento que se promueven- tenían ya relevancia el inicio del período estudiado.

En ese contexto estructural, la escuela ingresó al régimen de la ley SEP, donde se incorporó la Subvención y el PME como componente adicional de gestión escolar. El financiamiento de la SEP gatilló cambios en la capacidad potencial de la escuela y del sostenedor de financiar acciones que previamente no podía ejecutar.

A estos elementos deben incorporarse los condicionantes de tipo socioeconómico y geográfico, para completar una panorámica de los elementos que influenciaron la acción de los agentes. Ya se mencionó cuáles de los elementos de estos componentes favoreció o constriñó la acción. Ahora, siguiendo con la teoría de Archer (2009), será relevante observar la relación entre los componentes de las propiedades estructurales, considerando los elementos analíticos mencionados en el marco teórico, referidos a las diadas complementariedad/ incompatibilidad y necesario- contingente.

El caso descrito dio cuenta de una importante presencia de las relaciones de complementariedad de carácter necesario, las que se asocian a una mayor presencia 
de dinámicas morfoestáticas. Esto implica que las relaciones entre estas instituciones y entre estas y los condicionantes socioeconómicos, por lo general no sufrieron importantes cambios en el período analizado. Las relaciones complementarias y necesarias se han dado desde una lógica burocrática, donde el sostenedor y la escuela han intentado implementar las exigencias de las normas y políticas educativas. Es decir, los intereses del estado, del nivel local (sostenedor) y de la escuela, no se han visto en conflicto al entrar en interacción, ni se han modificado de manera relevante durante este período, al abordarse desde una lógica burocrática.

Sin embargo, del relato descrito del caso también es posible identificar la presencia de relaciones incompatibles y necesarias. En particular, los requerimientos y exigencias técnicas del sistema educativo hacia la escuela -dadas por la clasificación de la escuela o el desarrollo del PME-, no generaron sinergias relevantes con las dinámicas de la escuela, marcadas por el conflicto y la ausencia de un proyecto educativo claro para la comunidad. Este fenómeno estaba presente con anterioridad al período analizado.

Por su parte, la historia analizada no dio cuenta de relaciones relevantes de complementariedad y contingentes, así como de incompatibilidad y contingentes, siguiendo las definiciones de Archer, lo que da cuenta de la estabilidad de los procesos estructurales en el período analizado. La única excepción se observa en la gran crisis provocada por la huelga de los profesores, la que, sin perjuicio de ello, tampoco modificó el escenario general de la escuela. Mientras, los procesos necesarios, ya sean complementarios o incompatibles, terminan de completar un diagnóstico de ausencia de transformaciones en las propiedades del estrato de la estructura social, en el caso analizado.

\section{MORFOGÉNESIS DE LA CULTURA}

Respecto de los condicionantes culturales que estaban presentes desde etapas previas al período analizado, en primer lugar, pudo reconocerse la presencia de dos grandes lógicas de acción o estructuras de sentido para el sistema educativo. La primera de ellas es una cultura burocrática, en el sentido que la escuela se reconoce como parte de un sistema nacional, en el que tiene una función de implementar lo que la norma y política educacional define para los establecimientos públicos. La segunda, es una cultura del desempeño, en que está presente la valoración de la calidad de la escuela en base a los resultados que obtiene de diferente tipo de indicadores, especialmente de mediciones de aprendizaje (como Simce). En este caso, la segunda cultura no ha tenido mayor participación en la historia de la institución analizada, a diferencia de la primera tipología.

A estas tipologías, podría haberse agregado un énfasis especial del sostenedor respecto del sistema educativo local. Sin embargo, como el relato pudo evidenciar, este énfasis solo se visibilizó desde la perspectiva del control del gasto y decisiones administrativas de esa institución, mas no en un relato o proyecto pedagógico particular.

Asimismo, se identificaron "micro culturas" dentro del establecimiento, asociadas a la identidad y expectativas de los actores particulares que se desempeñan allí (equipo directivo, docentes, apoderados), las que convivían dificultosamente entre sí. 
Archer (2009) menciona que el proceso morfogenético de la cultura incluye cuatro configuraciones que apelan a la interacción entre sistemas de ideas que conviven en el análisis de un determinado fenómeno. Una de ellas se observó con mayor presencia en el caso analizado, la que se define como contradicciones constrictivas, que implica grados de tensión entre las ideas dominantes, con una tendencia mayor hacia la síntesis que a la ruptura. En el caso de estudio se evidenció una alta tensión de parte de las visiones de cada involucrado respecto de los demás, en cuanto a la visión de lo que la escuela necesita para mejorar. Esta contradicción no modificó de manera relevante la percepción respecto de la escuela, ni tampoco la relevancia relativa de las ideas en ese caso, manteniéndose la primacía de los requerimientos del sistema educativo nacional, considerando un perfil más burocrático que técnico.

En la teoría morfogenética, esta configuración en la interacción entre ideas está vinculada con los procesos morfoestáticos, al igual que lo que se distinguió en el caso analizado.

\section{HACIA LA INTEGRACIÓN DEL ANÁLISIS DEL PROCESO MORFOGENÉTICO: NIVEL POSICIONAL DE LOS INVOLUCRADOS}

De acuerdo con la teoría de Archer $(2000,2009)$, un factor causal del proceso morfogenético es el nivel posicional de los involucrados, es decir, el margen de agencia dada por las condiciones objetivas de vida y la predisposición que éstas generan hacia ciertas definiciones y acciones.

Si se evalúa el nivel posicional de las instituciones y agentes involucrados, y su efecto en la acción de mejoramiento de la escuela, se observa que las instituciones y agentes mantuvieron su mayor nivel posicional a lo largo del período estudiando, considerando la primacía que ejercer el Ministerio de Educación y el Sostenedor. Este nivel posicional estuvo relacionado con la jerarquía de estos organismos y la concentración de recursos. Claramente, ambos aspectos les entregaron mayor influencia que el resto de las instituciones y agentes involucrados, lo que explica la relación vertical con que estas instituciones y sus agentes se vinculan con los demás. No se observó tampoco que existiera una oposición o contradicción entre ambas instituciones.

Como contraparte, la escuela, y especialmente el equipo directivo, no tuvieron nivel posicional, ni manejaron recursos para ordenar su propio funcionamiento. $\mathrm{Al}$ contrario, se vieron impotentes frente a las determinaciones descritas por las instituciones que mantuvieron su mayor nivel posicional.

\section{INTERESES CREADOS Y RELACIÓN CON ESTABILIDAD Y CAMBIO}

De acuerdo con la teoría morfogenética, los agentes operan en un marco de condicionamiento estructural, lo que no compromete los poderes autónomos de su reflexividad. Sin embargo, este condicionamiento orienta la acción y en caso de no seguir esa ruta, los agentes deben asumir los costos. El condicionamiento estructural, al estar vinculado con la posición social de los agentes y la distribución de recursos, desarrolla lo que Archer denomina intereses creados, es decir, recursos que se poseen de manera desigual y que son la forma en que la estructura ejerce su influencia en las próximas acciones. 
Respecto de los casos de estudio, de lo que ha podido analizarse, los intereses creados que operaron con mayor relevancia fueron los del Sostenedor. Sus intereses creados les condujeron a supervigilar el funcionamiento de los procesos definidos por la normativa y dirigir su relación con la escuela. En este caso, el sostenedor no optó por desafiar sus intereses creados, sino más bien, mantuvo su perfil, vinculado con una posición orientada hacia mantener el control de los procesos de la institución.

Por su parte, el equipo directivo de la escuela buscó modificar su posición durante el período analizado. Sin embargo, no terminó desafiando sus intereses creados, por lo que actuó principalmente respecto del cumplimiento de las políticas y obligaciones legales asumidas con el sistema educativo nacional y comunal, la toma de decisiones sobre la gestión del establecimiento y el control interno.

Pese a ello, se identificaron períodos en que el equipo directivo buscó desafiar sus intereses creados, buscando modificar la dinámica interna y reordenar el funcionamiento de la institución. Sin embargo, este esfuerzo fracasó, evidenciándose serios costos para la directora del establecimiento, que terminó perdiendo legitimidad dentro del establecimiento.

\section{CONCLUSIONES}

El caso que acaba de analizarse evidenció la ausencia de procesos de morfogénesis, puesto que, no se identificaron cambios relevantes en los estratos de agencia, estructura social y cultural y en el conjunto de las interrelaciones entre esos estratos. Además de la ausencia de transformaciones, operó una suerte de anarquía entre las lógicas y sentidos ideas de las instituciones y personas involucradas, que estuvieron en permanente tensión, pero no desafiaron la distribución estructural del poder y de los recursos.

A nivel de agencia, se vislumbró también una debilidad en la constitución de agentes e incluso de roles dentro de la escuela, lo que tampoco se modificó, e incluso podría decirse que empeoró durante el período analizado.

Lo anterior se traduce en un panorama generalizado de estabilidad en la vinculación entre los factores analizados y la dinámica de los procesos de la escuela. Vale decir, el producto de la interacción entre los condicionantes estructurales y culturales y la agencia, en el contexto de funcionamiento de la escuela, no generó procesos de mejoramiento educativo.

La literatura sobre mejoramiento educativo ha tendido a identificar como "palancas" de los cambios positivos en las instituciones el liderazgo escolar -a nivel de sistema y dentro de la escuela- y su vínculo con la presencia y uso adecuado de recursos, así como de desarrollo de capacidades internas para la mejora (Harris, 2012). Ninguno de estos aspectos pudo desarrollarse exitosamente en el período analizado de la escuela presentada. Al contrario, cada uno de estos aspectos se identificó como una carencia, que condicionó negativamente los procesos y logros de la institución.

Por su parte, la literatura también ha destacado la influencia del contexto socioeconómico y cultural en el desempeño de una escuela. En el caso descrito, se ha identificado este fenómeno con particular relevancia, asociado a las bajas 
expectativas respecto de la formación que ofrece la escuela, la ausencia de un "foco pedagógico", o proyecto educativo, y la presencia de diversos focos de conflicto en la relación entre los miembros de la comunidad. Estos aspectos, han tendido en este caso a aportar a mantener la lógica de funcionamiento de la institución, en el período analizado.

Los resultados de esta investigación ayudan a verificar la visión de la literatura respecto de la dinámica del mejoramiento educativo. Junto con ello, la implementación del enfoque morfogenético favorece la identificación no solo de la multiplicidad de factores involucrados en un proceso de mejoramiento, sino también de las relaciones que se establecen entre estos elementos y las implicancias que ello tiene en el resultado esperado de la mejora educativa. Esto fue particularmente evidente al visibilizar la alta cantidad de fenómenos intervinientes en el proceso de implementación que ocurren a nivel meso (sostenedor) o micro (escuela y sus involucrados), la alta inestabilidad de los procesos que se generan en estos escenarios y la influencia que en ello tiene la cercanía entre la institución y la agencia, o la relevancia de los factores culturales en la instalación o en el rechazo a la presencia de procesos de mejoramiento.

Asimismo, la identificación del rol de la agencia y su margen de maniobra, en función de los condicionamientos estructurales y culturales, es una medida visible de la dificultad de los cambios necesarios para la mejora escolar, lo que permite dar claridad sobre el rol insustituible del sistema educativo en el proceso de mejora de una institución. No dependerá únicamente de los esfuerzos de esta su propia mejora, sino que esos esfuerzos estarán mediados por los apoyos y constreñimientos que ofrezca el sistema y no podrían escindirse del contexto histórico y sociocultural en que se desarrollan las instituciones educativas.

Los procesos de mejoramiento, al depender de diferentes instituciones, organismos y prácticas, tienen incidencia directa o indirecta en el proceso de mejoramiento y estas múltiples transacciones tardan tiempo. Esto implica que -tal como otros procesos de transformación social-no debiera necesariamente esperarse que las acciones orientadas hacia el mejoramiento educativo puedan avanzar hacia una etapa similar a la elaboración estructural de Archer, en el corto plazo. Esta observación es de gran relevancia, considerando la influencia que tienen, a nivel internacional y en Chile en particular, las políticas de "aseguramiento de la calidad", o Reformas Basadas en Estándares (Anstorp, 2010; Banco Mundial,2007), en que se espera que la estructura de incentivos y recursos dispuestos desde el estado central, redunden en una mejora sostenida y en el corto plazo de parte de las escuelas.

Considerando lo anterior, desde la aproximación morfogenética, fue particularmente evidente que el efecto esperado de políticas, mecanismos o incluso recursos financieros cuantiosos puede tener una incidencia menor a la esperada en el proceso de mejoramiento, lo que es diferente a la expectativa de un Sistema de Aseguramiento de la Calidad, que espera tener mayor control sobre sus resultados.

Todos estos elementos aportan a la comprensión que la mejora escolar puede ocurrir en procesos temporales que son diferentes -más largos-a las definiciones de una política, y que el horizonte de 5 años para lograr la efectividad -como el dispuesto por la política que se consideró para esta investigación- puede ser muy reducido. 
Esta situación implica que la política debiera profundizar en el análisis más pormenorizado de los plazos que define para las escuelas, así como en una definición más específica y contextualizada de lo que les pide.

\section{REFERENCIAS}

AGENCIA DE CALIDAD DE LA EDUCACIÓN. Manual para establecimientos. Chile: Agencia de Calidad de la Educación, 2014. Disponible en: http://archivos. agenciaeducacion.cl/biblioteca_digital_historica/orientacion/2014/manualest_2014. pdf. Acceso en el: 8 abr. 2018.

AGUILAR, O. Agencia, estructura y la emergencia del habitus. In: CHARRY, C.; ROJAS, N. (org.). La era de los individuos: actores, política y teoría en la sociedad actual. Santiago: Lom, 2013. p. 107-134.

AGUILAR, O. La teoría del habitus y la crítica realista al conflacionismo central. Persona y Sociedad, v. 22, n. 1, p. 9-26, 2008. https://doi.org/10.11565/pys.v22i1.155 ANDERSON, S. E. et al. No one way: differentiating school district leadership and support for school improvement. Journal of Educational Change, v. 13, p. 403-430, 2012. https://doi.org/10.1007/s10833-012-9189-y

ANSTORP, M. Using student test results for accountability and improvement: a literature review. Paris: OECD Education Working Paper, 2010.

ARCHER, M. Realismo e o problema da agência. Estudos de Sociologia, v. 2, n. 6, p. 51-75, 2000.

ARCHER, M. Making our way through the world: human reflexivity and social mobility. Cambridge: Cambridge University Press, 2007a.

ARCHER, M. The trajectory of the morphogenetic approach: An account in the firstperson. Sociologia, Problemas e Práticas, v. 54, p. 35-47, 2007 b.

ARCHER, M. Teoría social realista: el enfoque morfogenético. Santiago: Ediciones Universidad Alberto Hurtado, 2009.

ASOCIACIÓN DE MUNICIPALIDADES DE CHILE (AMUCH). Estudio de Seguridad Ciudadana y vulnerabilidad socio delictual de las 345 comunas del país. Santiago: AMUCH, 2014.

BANCO MUNDIAL. El diseño institucional de un sistema efectivo de aseguramiento de la calidad de la educación en Chile. Santiago: Banco Mundial, Oficina Regional para América Latina y el Caribe, 2007.

BELLEI, C. El estudio de la segregación socioeconómica y académica de la educación chilena. Estudios Pedagógicos, v. 39, n. 1, p. 325-345, 2013. http://dx.doi.org/10.4067/ S0718-07052013000100019

BELLEI, C.; VALENZUELA, J.; DE LOS RÍOS, D. Segregación escolar en Chile. In: MARTINIC, S.; ELACQUA, G. (org.). Cambios en la gobernanza del sistema educativo chileno. Santiago: UNESCO / P. Universidad Católica de Chile, 2010. p. 257-284. 
BELLEI, C. et al. Lo aprendí en la escuela. ¿Cómo se logran procesos de mejoramiento escolar? Santiago: Universidad de Chile / UNICEF, 2014.

BHASKAR, R. Realismo crítico, relaciones sociales y defensa del socialismo. Viento Sur, 2003. Disponible en: https://vientosur.info/realismo-critico-relaciones-socialesy-defensa-del-socialismo/. Acceso en el: 5 jun. 2014.

BOURDIEU, P. El sentido práctico. Madrid: Taurus, 1991.

ELMORE, R. Mejorando la escuela desde la sala de clases. Santiago: Fundación Chile, Fundación CAP, 2010.

FULLAN, M. Choosing the wrong drivers for whole system reform. Victoria: Centre for Strategic Education, 2011. Seminar Series Paper, No. 204. Disponible en: http://michaelfullan.ca/wp-content/uploads/2016/06/13396088160.pdf. Acceso en el: 6 jun. 2018.

FULLAN, M. Los nuevos significados del cambio en la educación. Barcelona: Octaedro, 2002.

FULLAN, M. The meaning of educational change. Nueva York: Teachers College Press, 1982.

GIDDENS, A. La constitución de la sociedad: bases para la teoría de la estructuración. Buenos Aires: Amorrurtú Editores, 1995.

HALLINGER, P.; HECK, R. Exploring the journey of school improvement: classifying and analyzing patterns of change in school improvement processes and learning outcomes. School Effectiveness and School Improvement. International Journal of Research, Policy and Practice, v. 22, n. 1, p. 1-27, 2011. https://doi.org/10.1080/092 43453.2010.536322

HARRIS, A. Liderazgo y desarrollo de capacidades en la escuela. Santiago: Fundación Chile, Fundación CAP, 2012.

HOPKINS, D. Hacia una buena escuela: experiencias y lecciones. Santiago: Fundación Chile, Fundación CAP, 2008.

LEVIN, B. Does politics help or hinder education change? Journal of Educational Change, v. 10, p. 69-72, 2009. https://doi.org/10.1007/s10833-008-9092-8

MACBEATH, J. Liderar el aprendizaje dentro y fuera de la escuela. Santiago: Fundación Chile, Fundación CAP, 2011.

MOURSHED, M.; CHIJIOKE, C.; BARBER, M. How the world's most improved school systems keep getting better. Londres: McKinsey and Company, 2010.

MURILLO, F. El movimiento teórico-práctico de mejora en la escuela. Algunas lecciones aprendidas para transformar los centros docentes. REICE, v. 1, n. 2, p. 1-22, 2003.

RINCÓN-GALLARDO, S.; FLEISCH, B. Bringing effective instructional practice to scale: An introduction. Journal of Educational Change, v. 17, p. 379-393, 2016. https://doi.org/10.1007/s10833-016-9288-2

SAYER, A. Method in social science: a realist approach. New York: Routledge, 1992. 
STRAUSS, A.; CORBIN,J. Basic of qualitative research: grounded theory procedures and techniques. California: Sage, 1990.

\section{SOBRE EL AUTOR}

Luis Felipe de la Vega es doctor en ciencias de la educación por la Pontificia Universidad Católica de Chile (Chile). Profesor e investigador del Centro de Estudios y Desarrollo de Educación Continua para la Docencia de la Universidad de Chile (Chile).

E-mail: luis.delavega@uchile.cl

Recibido el 24 de octubre de 2019 Aprobado el 20 de mayo de 2020 